\title{
Comparative proteomics of phloem exudates reveals long-distance signals potentially involved in Litchi chinensis flowering
}

\author{
L.-J. HUANG, H.-B. CHEN, Z.-Q. HU, X.-Y. LU, H.-Y. WANG, H. LIU, B.-Y ZHOU* \\ College of Horticulture, South China Agricultural University, Guangzhou 510642, P.R.China
}

\begin{abstract}
It is well known that phloem is essential for transporting proteins as long-distance signals. In this study, a proteomic approach was carried out to identify proteins accumulated in phloem exudates at the pre-floral induction (PFId) stage, at the floral induction (FId) stage, and at the floral initiation (FIn) stage. As a result, 45 phloem exudate proteins were detected. Six proteins were found at all three stages. Sixteen proteins were specific to the PFId stage, 14 proteins were specific to the FId and FIn stages. From the enriched gene ontology $(G O)$-terms related to transport, signaling, hormone, and development, 12 transport-related, 5 signaling-related, 10 hormone-related, and 9 development-related proteins were identified. It was found that arginine glycine glycine repeats nuclear RNA binding protein A (RGGA) was specific to the FId stage whereas heat shock protein 90-7, plasma membrane intrinsic protein 1-4, and the homolog protein encoded by At4g27190 were specific to the FIn stage. The relative abundances of sporulation protein F10D13.7 and ATP-dependent binding casette transporter $\mathrm{G}$ family member 37 were higher at the FId stage than at the PFId stage, and that of enolase 2 was higher at the FIn stage than at the PFId stage, suggesting that these phloem exudate proteins might act as long-distance signals involved in the transition from vegetative growth to reproductive growth in litchi. This study contributes to the comparative proteomic analysis of plant phloem sap, which will provide insights into proteins involved in floral induction as well as inter-organ communication during development in litchi.
\end{abstract}

Additional key words: floral induction, floral initiation, GO-terms, litchi, pre-floral induction.

The plant xylem performs the important function for transport of water and nutrient, as well as for long distance-signals (Notaguchi and Okamoto 2015). It is well known that the phloem is essential for transport of small molecules, phytohormones, peptides/proteins, and coding/ noncoding RNA (Lucas et al. 2013). These substances may play roles as long-distance signals produced from other tissues, and as media for crosstalk among organs, or between plants and environment.

Flowering is a transition from vegetative to the reproductive phase. In the model plant Arabidopsis, the floral stimulus is formed in the leaves. In the leaf tissues, CONSTANS (CO) activates FLOWERING LOCUS T (FT) (Samach et al. 2000), whose encoded protein is a long seeking florigen (Yang et al. 2007). The FT protein migrates from leaves through phloem to the apical meristem to promote floral initiation (Corbesier et al. 2007). In other plants such as cucurbits and rice, the FT protein or its analogues are proved to be long-distance florigenic signals also transported in the phloem (Lin et al. 2007, Tamaki et al. 2007).

Litchi is an evergreen fruit tree commercially cultivated in southern Asia, South Africa, and Australia. Floral initiation in litchi is triggered by low temperatures and enhanced by drought in autumn and winter (Menzel and Simpson 1988, Chen and Huang 2005, Zhou et al. 2014). Litchi trees grown in controlled growth chambers with stably low temperature can produce big and high quality panicles (Lu et al. 2017). Interestingly, litchi trees grown under high temperature conditions with only few

Submitted 17 June 2019, last revision 20 November 2019, accepted 20 December 2019.

Abbreviations: ENO2 - enolase 2; GO - gene ontology; PFId - pre-floral induction; FId - floral induction; FIn - floral initiation; HSP heat shock protein; PIP - plasma membrane intrinsic protein; RGGA - arginine glycine glycine repeats nuclear RNA binding protein A. Acknowledgements: The research results reported in this paper are funded by the National Natural Science Foundation of China (grants Nos. 31772249, 31572080), the National Litchi and Longan Research System (project No. CARS-32), the Innovation Team Project of the Department of Education of Guangdong Province (grant No. 2016KCXTD 011), and the Project of the Department of Education of Guangdong Province (2015KGJHZ 016).

* Corresponding author; fax: (+86) 20 85280228, e-mail: zhoubiyan@scau.edu.cn 
leaves treated with low temperature still could produce flowers, though the panicles were small (Zhang et al. 2017), suggesting that the low temperature treated leaves can produce flowering signals, which can be transported to apical meristem to activate flowering.

In this study, a proteomics approach was carried out to identify proteins accumulated in phloem exudates before floral induction as the vegetative stage, at floral induction stage, and at floral initiation stage as the reproductive stages. We compared the proteins in the phloem exudates with the aim to identify potential long-distance signals produced in leaves that can be transported through phloem to regulate flowering.

Fourteen-year-old litchi (Litchi chinensis Sonn.) cv. anyuehong trees were grown in the experimental orchard of South China Agricultural University (a latitude of $23^{\circ} 9^{\prime} 40^{\prime \prime} \mathrm{N}$, a longitude of $113^{\circ} 21^{\prime} 18^{\prime \prime} \mathrm{E}$ ). When the fruits were harvested, trees were trimmed to allow three flushes. When the last flushes (terminal shoots) were mature, the terminal shoots with similar phenological stage were selected. The shoots were collected on $1^{\text {st }}$ October as pre-floral induction (PFId) stage, $31^{\text {st }}$ October as floral induction (FId) stage, and $10^{\text {th }}$ November as floral initiation (FIn) stage in 2017. At the PFId stage, leaves of the terminal shoots were just mature and the buds were in dormant stage. At FId stage, the leaves became dark green and the buds were still in dormant stage. At the FIn stage, the buds sprouted and the panicle primordia could be found at the axil of the rudimentary leaves.

The terminal shoots in $6 \mathrm{~cm}$ length with two compound leaves were cut from the branches. Two leaflets in one compound leaves were kept. The cut shoots were re-cut in $20 \mathrm{mM} \mathrm{Na} \mathrm{NaDTA}_{2}$, and were placed in a microtube containing $2 \mathrm{~cm}^{3}$ of the $\mathrm{Na}_{2}$-EDTA solution, incubated for $2 \mathrm{~h}$. Then the solution was discarded. To remove all residual $\mathrm{Na}_{2}$ EDTA, the petioles of the cut shoots were washed with sterilized double-distilled water for 5 times. After that, the cut shoots were placed in a new microtube containing $1.5 \mathrm{~cm}^{3}$ of sterilized double-distilled water for exudate collection. All the cut shoots were incubated in boxes with humid atmosphere. The exudates were collected for $8 \mathrm{~h}$ according to the method discribed by Guelette et al. (2012). Ten microtubes of the exudates were pooled together and frozen in liquid nitrogen, and stored at $-80{ }^{\circ} \mathrm{C}$.

The phloem exudates were unfrozen at $4{ }^{\circ} \mathrm{C}$ and centrifuged at $12000 \mathrm{~g}$. To obtain pure protein, the phloem exudates were ultrafiltrated in $3 \mathrm{kD}$-ultrafiltration tube. The residue was washed using $100 \mathrm{mM}$ ammonium bicarbonate for three times. To the washed residue (about $100 \mathrm{~mm}^{3}$ ), dithiothreitol was added at a final concentration of $50 \mathrm{mM}$, and incubated at $56{ }^{\circ} \mathrm{C}$ for $30 \mathrm{~min}$. The iodoacetamide at a final concentration of $50 \mathrm{mM}$ was added and the solution was incubated in the dark at room temperature for $30 \mathrm{~min}$ and then under irradiance for $5 \mathrm{~min}$. The reacted solution was added with 5 times volume of acetone, kept at $-20{ }^{\circ} \mathrm{C}$ overnight for protein deposition, and centrifuged at $12000 \mathrm{~g}$ and $4{ }^{\circ} \mathrm{C}$ for $10 \mathrm{~min}$. The residue was dried in cupboard wind, added with trypsin, dissolved in $50 \mathrm{~mm}^{3}$ of $100 \mathrm{mM}$ ammonium bicarbonate. The mixture was incubated at $37{ }^{\circ} \mathrm{C}$ for $16 \mathrm{~h}$, and then lyophilized.

The samples were analyzed using Easy- $n L C$ nanoflow HPLC system connected to Orbitrap elite mass spectrometer (Thermo Fisher Scientific, San Jose, CA, USA). Solution A contained $0.1 \%(\mathrm{v} / \mathrm{v})$ formic acid in double distilled water. Solution B contained $0.1 \%(\mathrm{v} / \mathrm{v})$ formic acid in acetonitrile. The column $(R P-C 180.15 \times$ $150 \mathrm{~mm}$, Column Technology, Fremont, CA, USA) was equilibrated using $95 \%$ of solution A. Samples were loaded onto Thermo Scientific EASY column (two columns) by an autosampler and subjected to sequential separation by Thermo Scientific EASY trap column $(150 \mu \mathrm{m} \times 1.5 \mathrm{~cm}$, $5 \mu \mathrm{m}$ particle size, $0.1 \mathrm{~nm}$ pore size, $C 18)$ and analytical column $(75 \mu \mathrm{m} \times 25 \mathrm{~cm}, 5 \mu \mathrm{m}$ particle size, $0.1 \mathrm{~nm}$ pore size, C18) using a segmented $1 \mathrm{~h}$ gradient from solvent A to $50 \%$ solvent B for $50 \mathrm{~min}$, followed by $50-100 \%$ solvent $\mathrm{B}$ for $4 \mathrm{~min}$, and then $100 \%$ solvent $\mathrm{B}$ for $6 \mathrm{~min}$.

The mass spectrometer was operated in positive ion mode, and MS spectra were acquired over a range of $300-2000 \mathrm{~m} / \mathrm{z}$. The resolving powers of the MS scan and $\mathrm{MS} / \mathrm{MS}$ scan at $200 \mathrm{~m} / \mathrm{z}$ for the Orbitrap Elite were set as 70000 and 17500 , respectively. The top sixteen most intense signals in the acquired MS spectra were selected for further MS/MS analysis. The isolation window was $1 \mathrm{~m} / \mathrm{z}$, and ions were fragmented through higher energy collisional dissociation with normalized collision energies of $35 \mathrm{eV}$. The maximum ion injection time was set at $50 \mathrm{~ms}$ for the survey scan and $150 \mathrm{~ms}$ for the MS/MS scans, and the automatic gain control target values for full scan modes was set to $1.0 \times 10^{-6}$ and for MS/MS was $5 \times 10^{4}$. The dynamic exclusion duration was $30 \mathrm{~s}$.

The raw files were analyzed using the Proteome Discoverer v. 1.4 software (Thermo Fisher Scientific). Search for the fragmentation spectra was performed using the MASCOT search engine embedded in Proteome Discoverer against the self database fasta. The following search parameters were used: monoisotopic mass, trypsin as the cleavage enzyme, two missed cleavages, carbamidomethylation of cysteine as fixed modifications, and peptide charges of $2+, 3+$, and $4+$, and the oxidation of methionine, phospho (ST), GlyGly (K), acetyl (K), acetyl (N-term) was specified as variable modifications. The mass tolerance was set to $20 \mathrm{mg} \mathrm{dm}^{-3}$ for precursor ions and $0.1 \mathrm{Da}$ for the fragment ions. The results were filtered based on a false discovery rate (FDR) of no more than $1 \%$. Gene ontology $(G O)$ terms for the petides were obtained from a BLASTP search against the UniProtKB Swiss-Prot database (available online: http://www.uniprot. org/uniprot/). Enriched $G O$ terms were identified with the Fisher's exact test.

SDS-PAGE analysis and silver staining of the phloem exudates showed protein compositions at the three stages, suggesting the collected phloem exudates could be used to identify proteins by shotgun analysis. As a result of the shotgun analysis, we totally detected 45 proteins that were present in the phloem exudates (Table 1 Suppl.). Six proteins were found at the three stages, whereas 16 proteins were specific to the PFId stage, regarded as the vegetative stage, 14 proteins were at the FId and FIn stages, regarded as the reproductive stages (Fig. 1). 


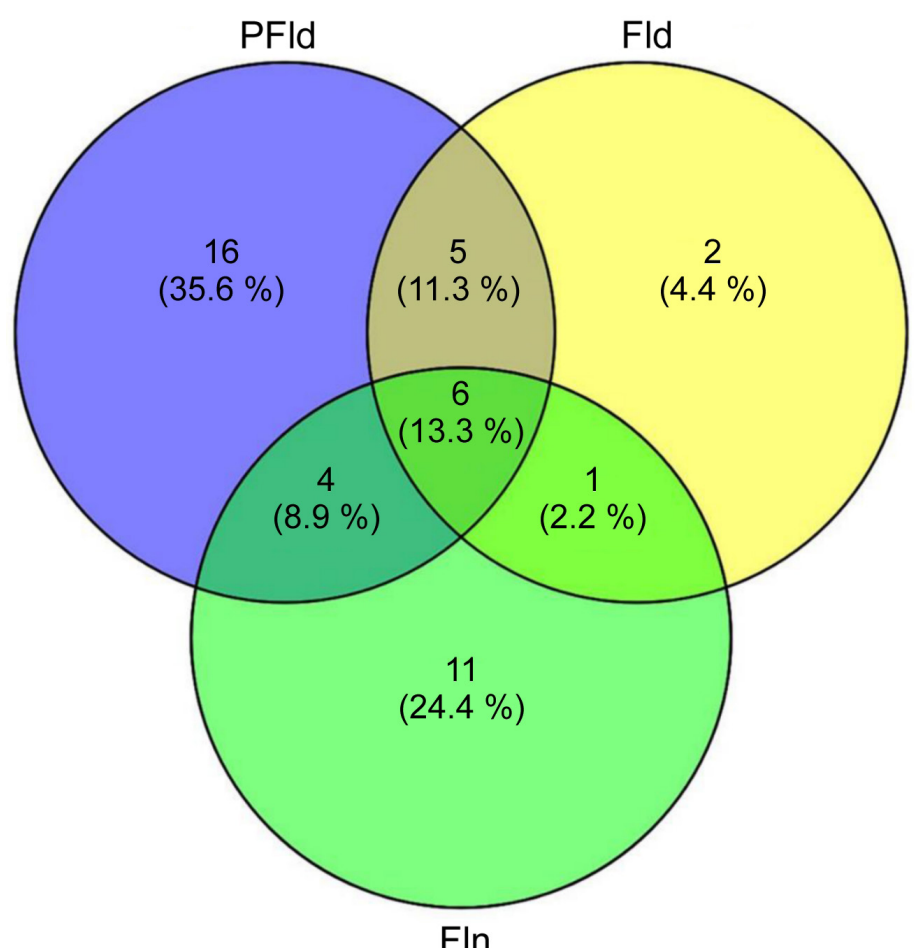

Fig. 1. A Venn diagram of the number of phloem exudate proteins at the pre-floral induction (PFId) stage, floral induction (FId) stage, and floral initiation (FIn) stage.

We then preformed $G O$-term analysis. At the PFId stage, the top three hit enriched $G O$-term of the biological processes were the catabolic process, cellular catabolic process, and reactive oxygen species metabolic process. For cell component, the top hit enriched $G O$-terms were the cytoplasm, intracellular membrane-bound organelle, and membrane-bound organelle. For molecular function, the $G O$-terms were ion binding, metal ion binding, and cation binding. At the FId stage, the top hit enriched $G O$-terms of the biological processes were response to stimulus, response to stress, and reactive oxygen species. For cell component, the top hit enriched $G O$-terms were the cytoplasm and cytoplasmic parts. For molecular function, tetrapyrrole binding is the top hit enriched $G O$-term. At the FIn stage, the top hit enriched $G O$-terms were response to stress and catabolic processes in the biological process, cytoplasmic parts in the cell component, and copper ion binding in the molecular function.

From the enriched transport-related $G O$-terms including transport, RNA transport, auxin transport, transmembrane transport, regulation of transporter activity, protein transport, auxin polar transport, we identified 12 transportrelated homologous proteins. As shown in Table 1 Suppl., they are L-ascorbate peroxidase (APX1), plastocyanin major isoform (DRT112), blue copper protein (BCB), ranbinding protein 1 homolog c (RANBP1C), peroxisomal (S)-2-hydroxy-acid oxidase (GLO5), ATP synthase subunit beta-3 (Atp3), triosphosphate isomerase (CTIMC), ATP synthase subunit alpha (ATPA), patellin-5 (PATL5), ABC transporter $\mathrm{G}$ family member 37 (ABCG37), probable aquaporin PIP1-4 (PIP1.4), and endoplasmin homolog (HSP90-7).
From the enriched signalling-related $G O$-terms including signal transduction, hormone-mediated signalling pathway, positive regulation of auxin mediated signalling pathway, we identified 5 signalling-related homologous proteins. They are peptidyl-prolyl cistransisomerase (CYP19-1), GLO5, serine/threonineprotein kinase (TOR), RGG repeats nuclear RNA binding protein A (RGGA), and At4g27190 (Table 1 Suppl.).

From the enriched hormone-related $G O$-terms including response to hormone, response to abscisic acid, response to auxin, regulation of hormone content, brassinosteroid biosynthetic process, we identified 10 hormone-related homologous proteins. They are APX1, $\mathrm{BCB}, \mathrm{CTIMC}$, bifunctional enolase 2 (ENO2), ABCG37, TOR, chlorophyll $a-b$ binding protein 2.2 (LHCB2.2), RGGA, acid phosphatase-like protein (ACP), and DRT112 (Table 1 Suppl.).

From the enriched development-related $G O$-terms including developmental process, reproductive system development, vegetative to reproductive phase transition of meristem, flower development, meristem development, regulation of meristem growth, we identified 9 hormonerelated homologous proteins. They are APX1, peroxidase 53 (PER53), DRT112, acidic endochitinase (CHIB1), sporulation protein (F10D13.7), ABCG37, TOR, midasin (AtMDN1), HSP90-7 (Table 1 Suppl.).

From the above phloem exudate proteins related to transport, signalling, hormone, and development, we identified those specific to the FId and FIn stages or those with a higher relative abundance at the FId and FIn stage compared to those at the PFId stage. We found that RGGA was specific to the FId stage, while HSP90-7, PIP1.4, 
and the homologous protein encoded by $A t 4 g 27190$ were specific to the FIn stage. We also found that the relative abundance of sporulation protein F10D13.7 and ABCG37 was higher at the FId stage than at the PFId stage, and that amounts of ENO2 was higher at the FIn stage than at the PFId stage.

The plant phloem system provides an avenue for transport of proteins which may act as long distancesignals (Notaguchi and Okamoto 2015). Comparative proteomics analysis of phloem sap from resistant and susceptible rice cultivars has been carried out to investigate long-distance signalling (Du et al. 2015). Phloem exudates of Arabidopsis thaliana during the induction of systemic acquired resistance (SAR) with virulent or avirulent strains of Pseudomonas syringae has been performed to identified proteins contribute to the SAR response (Carella et al. 2016). Also, a study of phloem exudates collected from salt-stressed cucumber has been done to identify salt-responsive phloem proteins (Fan et al. 2015). These comparative proteomics studies are focused on biotic and abiotic stress responses.

Flowering, an important developmental stage for plants. Interestingly, it has been proven that the flowering signals are produced in leaves and transmitted through phloem system (Corbesier et al. 2007). In leaves, FT is activated by $\mathrm{CO}$ and then the FT protein in Arabidopsis and its analogue $\mathrm{Hd} 3 \mathrm{a}$ in rice migrate through phloem to the apical meristem to promote floral initiation (Lin et al. 2007, Tamaki et al. 2007). Aki et al. (2008) performed a shotgun analysis of the proteome of phloem saps from rice, and found the presence of three TERMINAL FLOWER 1 proteins in phloem sap, suggesting that apart from the FT protein, other flowering-related proteins may exist in the phloem sap and act as long-distance signals. However, much less comparative proteomic studies on floralinductive proteins in phloem exudates are reported than those on the stress-responsive proteins. Up till now, no publication has reported related studies on floral-inductive proteins in the litchi phloem exudates.

Litchi is an evergreen fruit tree requiring low temperature for floral induction (Chen and Huang 2005, $\mathrm{Lu}$ et al. 2017). However, litchi floral induction always conducts now under warm winter condition which is attributed to global warming, causing poor litchi flowering. It is important to reveal the genetic control of flowering so as to find methods to bypass chilling. One of these tasks is to find out the flowering-related proteins that may act as long-distance signals in the phloem saps. In our study, we performed shotgun analysis and detected 45 proteins that were present in the phloem exudates. From the phloem exudate proteins related to transport, signalling, hormone, and development, we further screened those specific to the FId and FIn stages, the reproductive stages, or those with a higher relative abundance at the FId and FIn stages compared to those at the PFId vegetative stage. As a result, we identified RGGA, HSP90-4, PIP1.4, a homologous protein encoded by At4g27190, F10D13.7, ABCG37, and ENO2.

The RGGA belonged to the $G O$-terms including the signal transduction (GO:0007165), hormone-mediated signalling pathway (GO:0009755), in the response to hormone (GO:0009725), and response to abscisic acid (GO:0009737). It is a nucleus-localized RNA-binding protein, containing arginine-rich regions with several RGG repeats at their $\mathrm{N}$ - and $\mathrm{C}$-termini (Landsberger et al. 2002). HSP90-7 is regarded to function in the protein transport (GO:0015031) and regulation of meristem growth (GO:0010075). Heat-shock protein 90 (HSP90) is a highly conserved molecular chaperone that is involved in modulating a multitude of cellular processes under both physiological and stress conditions (Chong et al. 2015). It can fold protein kinases and steroid hormone receptors (Richter and Buchner 2001, Young et al. 2001). HSP90-7 is an ER-localized HSP90 paralogue regarded as the HSP90B subfamily (Chen et al. 2006). The mutant in which a T-DNA is inserted in the promoter region of HSP90-7, has an enlarged dome-shaped shoot apical meristem (Ishiguro et al. 2002). The PIP1.4 belonged to the transmembrane transport (GO:0055085); it is a member of the plasma membrane intrinsic proteins. Constitutive overexpression of PIP1;4 promotes sap flow and water transport under cold stress (Jang et al. 2007). In this study, our result showed that RGGA, HSP90-7, and PIP1.4 were specific to the FId or FIn stages suggesting that these phloem exudate proteins might act as long-distance signals involved in the transition from vegetative growth to reproductive growth.

The sporulation protein F10D13.7 belonged to the $G O$ terms including the developmental process (GO:0032502), meristem development (GO:0048507), and regulation of meristem growth (GO:0010075). It plays a role in the regulation of root meristem cell division (Zhou et al. 2011). ABCG37 functions in a wide range of biological processes including transport, hormone, and development. It is an ATP-binding cassette (ABC)-type transporter (Ziegler et al. 2017); it transports the endogenous auxin precursor indole-3-butyric acid (Ruzicka et al. 2010). The ENO2 belonged to the $G O$-terms response to hormone (GO:0009725) and response to abscisic acid (GO:0009737). It is also called LOS2, and can bind to the promoter of STZ/ZAT10 from Arabidopsis. It has a direct regulatory function in controlling $S T Z / Z A T 10$ under low temperature (Lee et al. 2002). The low temperature is also needed for litchi floral induction. In the present study, we found that relative abundance of sporulation protein F10D13.7 and ABCG37 was higher at the FId stage than at the PFId stage, and that abundance of ENO2 was higher at the FIn stage than at the the PFId stage, suggesting that these phloem exudate proteins might act as long-distance signals involved in the transition from vegetative growth to reproductive growth.

The FT protein in Arabidopsis and its analogue Hd3a in rice are proved to be long-distance signals migrating through phloem to the apical meristem to promote floral initiation. However, we did not find the homologous protein FT in the phloem exudate of litchi. Aki et al. (2008) performed a shotgun analysis of the proteome of phloem sap from rice. They did not find the FT analogue Hd3a, but the presence of three TERMINAL FLOWER 1 proteins. It may be that the amount of this protein was too low to be determined. It may also be that other proteins but not 
the FT might act as long-distance signals controlling floral induction in litchi. Further studies should be carried out to identify the florigen, and the functional studies should be performed for the identification of phloem exudate proteins in litchi.

In conclusion, our results provide the first insights into long-distance proteins involved in floral induction as well as inter-organ communication during development in litchi.

\section{References}

Aki, T., Shigyo, M., Nakano, R., Yoneyama, T., Yanagisawa, S.: Nano scale proteomics revealed the presence of regulatory proteins including three FT-like proteins in phloem and xylem saps from rice. - Plant Cell Physiol. 49: 767-790, 2008.

Carella, P., Merl-Pham, J., Wilson, D.C., Dey, S., Hauck, S., Vlot, A.C., Cameron, R K.: Comparative proteomics analysis of Arabidopsis phloem exudates collected during the induction of systemic acquired resistance. - Plant Physiol. 171: 1495$1510,2016$.

Chen. B., Zhong. D., Monteiro. A.: Comparative genomics and evolution of the HSP 90 family of genes across all kingdoms of organisms. - BMC Genomics 7: 156, 2006.

Chen, H.B., Huang, H.B.: Low temperature requirements for floral induction in lychee. - Acta hort. 665: 195-202, 2005.

Chong, L.P., Wang, Y., Gad, N., Anderson, N., Shah, B., Zhao, R.: A highly charged region in the middle domain of plant endoplasmic reticulum (ER)-localized heat-shock protein 90 is required for resistance to tunicamycin or high calciuminduced ER stresses. - J. exp. Bot. 66: 113-124, 2015.

Corbesier, L., Vincent, C., Jang, S., Fornara, F., Fan, Q., Searle, I., Giakountis A., Farrona, S., Gissot, L., Turnbull, C., Coupland, G.: FT protein movement contributes to long-distance signaling in floral induction of Arabidopsis. - Science 316: 1030-1033, 2007.

Du, B., Wei, Z., Wang, Z., Wang, X., Peng, X., Du, B., Chen, R., Zhu, L., He, G.: Phloem-exudate proteome analysis of response to insect brown plant-hopper in rice. - J. Plant Physiol. 183: 13-22, 2015.

Fan, H., Xu, Y., Du, C., Wu, X.: Phloem sap proteome studied by iTRAQ provides integrated insight into salinity response mechanisms in cucumber plants. - J. Proteomics 125: 54-67, 2015.

Guelette, B.S., Benning, U.F., Hoffmann-Benning, S.: Identification of lipids and lipid-binding proteins in phloem exudates from Arabidopsis thaliana. - J. exp. Bot. 63: $3603-$ 3616, 2012.

Ishiguro, S., Watanabe, Y., Ito, N., Nonaka, H., Takeda, N., Sakai, T., Kanaya, H., Okada, K.: SHEPHERD is the Arabidopsis GRP94 responsible for the formation of functional CLAVATA proteins. - EMBO J. 21: 898-908, 2002.

Jang, J.Y., Lee, S.H., Rhee, J.Y., Chung, G.C., Ahn, S.J., Kang, H.: Transgenic Arabidopsis and tobacco plants overexpressing an aquaporin respond differently to various abiotic stresses. Plant mol. Biol. 64: 621-631, 2007.

Landsberger, M., Lorkovic, Z. J., Oelmuller, R.: Molecular characterization of nucleus-localized RNA-binding proteins from higher plants. - Plant mol. Biol. 48: 413-421, 2002.

Lee, H., Guo, Y., Ohta, M., Xiong, L., Stevenson, B., Zhu, J.K.:
LOS2, a genetic locus required for cold-responsive gene transcription encodes a bi-functional enolase. - EMBO J. 21: 2692-2702, 2002.

Lin, M., Belanger, H., Lee, Y.J., Varkonyi-Gasic, E., Taoka, K.I., Miura, E., Xoconostle-Cazares, B., Gendler, K., Jorgensen, R.A., Phinney, B., Lough, T.J., Lucas, W.J.: FLOWERING LOCUS $\mathrm{T}$ protein may act as the long-distance florigenic signal in the cucurbits. - Plant Cell 19:1488-1506, 2007.

Lu, X., Li, J., Chen, H., Hu, J., Liu, P., Zhou B.: RNA-seq analysis of apical meristem reveals integrative regulatory network of ROS and chilling potentially related to flowering in Litchi chinensis. - Sci. Rep. 7: 10183, 2017.

Lucas, W. J., Groover, A., Lichtenberger, R., Furuta, K., Yadav, S. R., Helariutta, Y., He, X. Q., Fukuda, H., Kang, J., Brady, S.M., Patrick, J.W., Sperry, J., Yoshida, A., López-Millán, A.F., Grusak, M.A., Kachroo, P.: The plant vascular system: evolution, development and functions. - J. integr. Plant Biol. 55: 294-388, 2013.

Menzel, C.M., Simpson, D.R.: Effect of temperature on growth and flowering of litchi (Litchi chinensis Sonn.) cultivars. - J. hort. Sci. 63: 349-360, 1988.

Notaguchi, M., Okamoto, S.: Dynamics of long-distance signaling via plant vascular tissues. - Front. Plant Sci. 6: 161, 2015.

Richter, K., Buchner, J.: Hsp90: chaperoning signal transduction. - J. cell. Physiol. 188: 281-290, 2001.

Ruzicka, K., Strader, L.C., Bailly, A., Yang, H., Blakeslee, J., Langowski, L., Nejedla, E., Fujita, H., Itoh, H., Syono, K., Hejátko, J., Gray, W.M., Martinoia, E., Geisler, M., Bartel, B., Murphy, A.S., Friml, J.: Arabidopsis PIS1 encodes the ABCG37 transporter of auxinic compounds including the auxin precursor indole-3-butyric acid. - Proc. Nat. Acad. Sci. 107: 10749-10753, 2010.

Samach, A., Onouchi, H., Gold, S.E., Ditta, G.S., SchwarzSommer, Z., Yanofsky, M.F., Coupland, G.: Distinct roles of CONSTANS target genes in reproductive development of Arabidopsis. - Science 288: 1613-1616, 2000.

Tamaki, S., Matsuo, S., Wong, H.L., Yokoi, S.; Shimamoto, K.: Hd3a protein is a mobile flowering signal in rice. - Science 316:1033-1036, 2000.

Yang, Y., Klejnot, J., Yu, X., Liu, X., Lin, C.: Florigen (II): it is a mobile protein. - J. Integr. Plant Biol. 49: 1665-1669, 2007.

Young, J. C., Moarefi, I., Hartl, F.U.: Hsp90: a specialized but essential protein-folding tool. - J. cell. Biol. 154: 267-273, 2001.

Zhang, H., Shen, J., Wei, Y., Chen, H.: Transcriptome profiling of litchi leaves in response to low temperature reveals candidate regulatory genes and key metabolic events during floral induction. - BMC Genomics 18: 363, 2017.

Zhou, B.Y., Chen, H.B., Wu, C.B.: An overview on natural triggers and stress signals in relation to flowering in Litchi chinensis and Dimocarpus longan. - Acta hort. 1029: 137144, 2014.

Zhou, X., Li, Q., Chen, X., Liu, J., Zhang, Q., Liu, Y., Liu, K., $\mathrm{Xu}$, J.: The Arabidopsis RETARDED ROOT GROWTH gene encodes a mitochondria-localized protein that is required for cell division in the root meristem. - Plant Physiol. 157: 17931804, 2011.

Ziegler, J., Schmidt, S., Strehmel, N., Scheel, D., Abel, S.: Arabidopsis transporter ABCG37/PDR9 contributes primarily highly oxygenated coumarins to root exudation. - Sci. Rep. 7: 3704, 2017. 